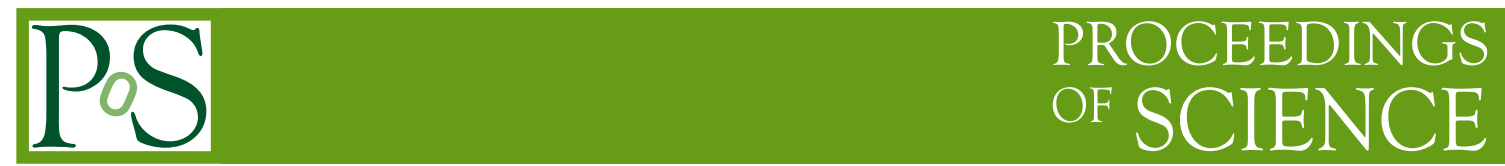

\title{
Scalar Mesons at BESIII
}

\section{Guangming $\mathbf{X u}^{* \dagger}$}

Peking University

E-mail: xugmepku.edu.cn

The preliminary results from the partial wave analyses of $J / \psi \rightarrow \gamma \omega \phi, \gamma \pi^{0} \pi^{0}, \gamma \eta \eta$ are reported, based on $225 \mathrm{M} J / \psi$ events collected with the BESIII detector at BEPCII. A $0^{++}$state, which was observed at BESII in the $\omega \phi$ mass spectrum in $J / \psi \rightarrow \gamma \omega \phi$, is confirmed at BESIII. The scalars $f_{0}(1500)$ and $f_{0}(1710)$ are observed and measured in both $J / \psi \rightarrow \gamma \eta \eta$ and $\gamma \pi^{0} \pi^{0}$ decays.

Xth Quark Confinement and the Hadron Spectrum,

October 8-12, 2012

TUM Campus Garching, Munich, Germany

*Speaker.

$\dagger$ On behalf of the BESIII Collaboration. 


\section{Introduction}

Our present understanding of the strong interaction is based on a non-Abelian gauge field theory, Quantum Chromodynamics (QCD), which describes the interactions of quarks and gluons; it also predicts the existence of new types of hadrons with explicit gluonic degrees of freedom (eg. glueballs, hybrids and multi-quarks) [1, 2, 3, 4, 5]. These unconventional states, if they exist, will enrich the meson spectroscopy greatly and shed light on the dynamics of QCD. According to lattice QCD predictions [6, 7], the lowest mass glueball with $J^{P C}=0^{++}$is in the mass region from 1.5 to $1.7 \mathrm{GeV} / c^{2}$. Although the identification of glueball is complicated due to the mixing of the pure glueball with nearby $q \bar{q}$ nonet, several glueball candidates are still found in the experiments. The abundance of scalar mesons in the this mass region, i.e. the $f_{0}(1500)[8,9,10,11,12,13]$, $f_{0}(1710)[11,12,14,15]$ and $X(1810)$ [16] makes them natural scalar glueball candidates. Radiative $J / \psi$ decay is a gluon-rich process and has long been regarded as one of the most promising hunting grounds for glueballs.

BEPCII/BESIII [17] is an upgrade facility from the previous BEPC/BESII experiment. The BESIII spectrometer consists of a main drift chamber with momentum resolution $0.5 \%$ at $1 \mathrm{GeV} / c$, a time-of-flight counter, a superconducting magnet with a magnetic strength of $1 \mathrm{~T}$, and a muon chamber system made of resistive plate chambers. Based on a sample of $2.25 \times 10^{8} \mathrm{~J} / \psi$ events [18] taken in 2009, the light hadron spectroscopy is widely studied.

\section{PWA of $J / \psi \rightarrow \gamma \eta \eta$}

An early study of $J / \psi \rightarrow \gamma \eta \eta$ was made by the Crystal Ball Collaboration [19] with the first observation of $f_{0}(1710)$, but the study suffered from low statistics. The $\eta \eta$ system has also been widely studied using data from $\bar{p} p$ annihilation [20, 21, 22, 23, 24, 15], as well as the data from $\pi^{-} p$ interaction [25].

Based on the $225 \mathrm{M} J / \psi$ events collected with the BESIII detector, The process $J / \psi \rightarrow$ $\gamma \eta \eta, \eta \rightarrow \gamma \gamma$ has been studied [26]. Clear diagonal bands, which correspond to the structures observed in the $\eta \eta$ invariant mass spectrum, can be seen in the Dalitz plot for the selected $J / \psi \rightarrow$ $\gamma \eta \eta$ candidate events (Fig. 1 (a)). To reject the $J / \psi \rightarrow \phi \eta(\phi \rightarrow \gamma \eta)$ events, we further require $\left|M_{\gamma \eta}-m_{\phi}\right|>0.03 \mathrm{GeV} / c^{2}$. Fig. 1 (b) shows the $\eta \eta$ invariant mass spectrum of the surviving events.

With GPUPWA, a Partial Wave Analysis (PWA) framework harnessing GPU parallel computing [27], a PWA was performed to disentangle the structures present in $J / \psi \rightarrow \gamma \eta \eta$ decays. The quasi two-body decay amplitudes in the sequential decay process $J / \psi \rightarrow \gamma X, X \rightarrow \eta \eta$ are constructed using covariant tensor amplitudes described in Ref. [28].

There are six resonances, $f_{0}(1500), f_{0}(1710), f_{0}(2100), f_{2}^{\prime}(1525), f_{2}(1810), f_{2}(2340)$ as well as $0^{++}$phase space and $J / \psi \rightarrow \phi \eta$ included in the basic solution. The masses and widths of the resonances, branching ratios of $J / \psi$ radiative decaying to $X$ and the statistical significances are summarized in Table 1

The dominant scalar components are from $f_{0}(1710)$ and $f_{0}(2100)$. For the $f_{0}(1710)$ meson, the PWA gives a mass of $1759 \pm 6 \mathrm{MeV} / c^{2}$ and a width of $172 \pm 10 \mathrm{MeV} / c^{2}$ with a statistical significance of $25 \sigma$; the mass and width are consistent with those obtained from $J / \psi \rightarrow \gamma K \bar{K}[11]$ and 

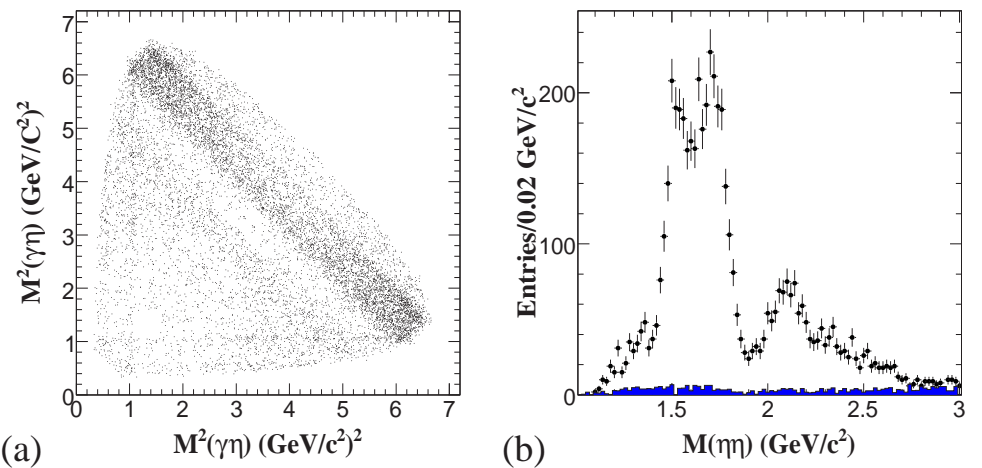

Figure 1: (a) Dalitz plot (two entries/event), before $\phi \rightarrow \gamma \eta$ veto. (b) The invariant mass spectrum of $\eta \eta$. The dots with error bars are data, and the shaded histogram is background estimated from $\eta$ sidebands.

$J / \psi \rightarrow \gamma \pi \pi[12]$ at BESII. The $f_{0}(2100)$ is observed with a statistical significance of $13.9 \sigma$, and its mass and width are determined to be $2081 \pm 13 \mathrm{MeV} / c^{2}$ and $273_{-24}^{+27} \mathrm{MeV} / c^{2}$, respectively, which are in agreement with previous measurements [10, 20, 21, 22]. The $f_{0}(1500)$ is observed with a statistical significance of $8.2 \sigma$, but its production rate, $\mathscr{B}\left(J / \psi \rightarrow \gamma f_{0}(1500) \rightarrow \gamma \eta \eta\right)=\left(1.65_{-0.31}^{+0.26}\right) \times 10^{-5}$, is about one order of magnitude lower than that of $f_{0}(1710)$ and $f_{0}(2100)$ since its dominant decay modes are $4 \pi$ and $\pi \pi[29]$. The mass and width obtained from the global fit are $1468_{-15}^{+14} \mathrm{MeV} / \mathrm{c}^{2}$ and $136_{-26}^{+41} \mathrm{MeV} / c^{2}$, respectively, which are consistent with the BESII measurements in $J / \psi \rightarrow$ $\gamma \pi \pi[12]$.

Table 1: Summary of the PWA results, including the masses and widths for resonances, branching ratios of $J / \psi \rightarrow \gamma \mathrm{X}$, as well as the significance. The first errors are statistical and the second ones are systematic. The statistic significances here are obtained according to the changes of the log likelihood.

\begin{tabular}{|c|c|c|c|c|}
\hline Resonance & Mass $\left(\mathrm{MeV} / c^{2}\right)$ & Width $\left(\mathrm{MeV} / \mathrm{c}^{2}\right)$ & $\mathscr{B}(J / \psi \rightarrow \gamma X \rightarrow \gamma \eta \eta)$ & Significance \\
\hline$f_{0}(1500)$ & $1468_{-15-74}^{+14+23}$ & $136_{-26-100}^{+41+28}$ & $\left(1.65_{-0.31-1.40}^{+0.26+0.51}\right) \times 10^{-5}$ & $8.2 \sigma$ \\
\hline$f_{0}(1710)$ & $1759 \pm 6_{-25}^{+14}$ & $172 \pm 10_{-16}^{+32}$ & $\left(2.35_{-0.11-0.74}^{+0.13+1.24}\right) \times 10^{-4}$ & $25.0 \sigma$ \\
\hline$f_{0}(2100)$ & $2081 \pm 13_{-36}^{+24}$ & $273_{-24-23}^{+27+70}$ & $\left(1.13_{-0.10-0.28}^{+0.09+0.64}\right) \times 10^{-4}$ & $13.9 \sigma$ \\
\hline$f_{2}^{\prime}(1525)$ & $1513 \pm 5_{-10}^{+4}$ & $75_{-10-8}^{+12+16}$ & $\left(3.42_{-0.51-1.30}^{+0.43+1.37}\right) \times 10^{-5}$ & $11.0 \sigma$ \\
\hline$f_{2}(1810)$ & $1822_{-24-57}^{+29+66}$ & $229_{-42-155}^{+52+88}$ & $\left(5.40_{-0.67-2.35}^{+0.60+3.42}\right) \times 10^{-5}$ & $6.4 \sigma$ \\
\hline$f_{2}(2340)$ & $2362_{-30-63}^{+31+140}$ & $334_{-54-100}^{+62+165}$ & $\left(5.60_{-0.65-2.07}^{+0.62+2.37}\right) \times 10^{-5}$ & $7.6 \sigma$ \\
\hline
\end{tabular}

\section{PWA of $J / \psi \rightarrow \gamma \pi^{0} \pi^{0}$}

The $J / \psi \rightarrow \gamma \pi^{+} \pi^{-}$process was analyzed previously in the MarkIII [30], DM2 [31], and BESI [32] experiments, the $J / \psi \rightarrow \gamma \pi^{0} \pi^{0}$ process was also studied by the Crystal Ball [33] and BESI [34] experiments, but no partial wave analysis was performed.

The analysis of $J / \psi \rightarrow \gamma \pi^{+} \pi^{-}$and $J / \psi \rightarrow \gamma \pi^{0} \pi^{0}$ was performed based on the $58 \mathrm{M} J / \psi$ events collected with BESII [12]. PWA was carried out using covariant tensor amplitude method only in the 1.0 to $2.3 \mathrm{GeV} / c^{2} \pi \pi$ mass range, due to background and statistic considerations. There are conspicuous peaks due to the $f_{2}(1270)$ and two $0^{++}$states in the 1.45 and $1.75 \mathrm{GeV} / c^{2}$ mass 
regions, identified as $f_{0}(1500)$ and $f_{0}(1710)$, respectively. However, more experimental data are needed to clarify the properties of these scalar states.

Considering the numerous backgrounds of $J / \psi \rightarrow \pi^{+} \pi^{-} \pi^{0}$ which dominates by $J / \psi \rightarrow \rho \pi$ for the process $J / \psi \rightarrow \gamma \pi^{+} \pi^{-}$, only the $J / \psi \rightarrow \gamma \pi^{0} \pi^{0}$ process is studied based on the $225 \mathrm{M} J / \psi$ events collected with the BESIII detector. The Dalitz plot is shown in Fig. 2 (a), where we can see clearly the bands corresponding to structures in $\pi^{0} \pi^{0}$ invariant mass spectrum. To reject the $J / \psi \rightarrow \omega \pi^{0} \rightarrow \gamma \pi^{0} \pi^{0}$ events, we further require $\left|M_{\gamma \pi^{0}}-m_{\omega}\right|>0.03 \mathrm{GeV} / c^{2}$. Fig. 2 (b) shows the $\pi^{0} \pi^{0}$ invariant mass spectrum of data and the backgrounds estimated from $\pi^{0}$ sidebands.
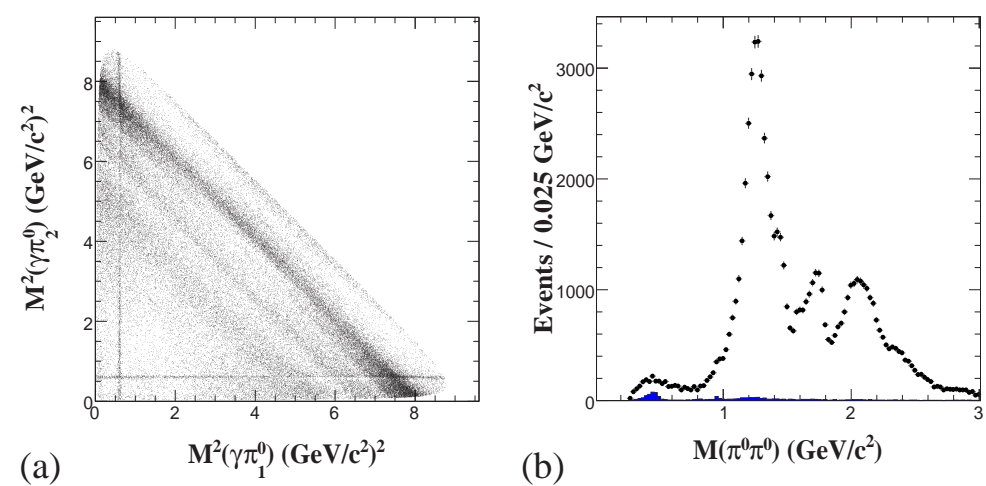

Figure 2: (a) The Dalitz plot, before $\omega \rightarrow \gamma \pi^{0}$ veto. (b) Invariant mass spectrum of $\pi^{0} \pi^{0}$. The histogram with error bars correspond to the data. The backgrounds estimated from $\pi^{0}$ sidebands are in shaded histogram.

With GPUPWA [27], PWA was performed to disentangle the structures present in $J / \psi \rightarrow$ $\gamma \pi^{0} \pi^{0}$ decays. The quasi two-body decay amplitudes in the sequential decay process $J / \psi \rightarrow$ $\gamma X, X \rightarrow \pi^{0} \pi^{0}$ are constructed using covariant tensor amplitudes described in Ref. [28]. The scalar resonances $f_{0}(500), f_{0}(1500), f_{0}(1710), f_{0}(2020)$ and tensor resonances $f_{2}(1270), f_{2}^{\prime}(1525)$, $f_{2}(1950), f_{2}(2150), f_{2}(2340)$ are found to be necessary in the basic solution of PWA, as well as the contribution from phase space and the process of $J / \psi \rightarrow \omega \pi^{0}$. The comparisons of the $\pi^{0} \pi^{0}$ invariant mass spectrum is displayed in Fig. 3, The dots with error bars are data (with background subtracted) and the solid histogram shows the PWA fit projection.

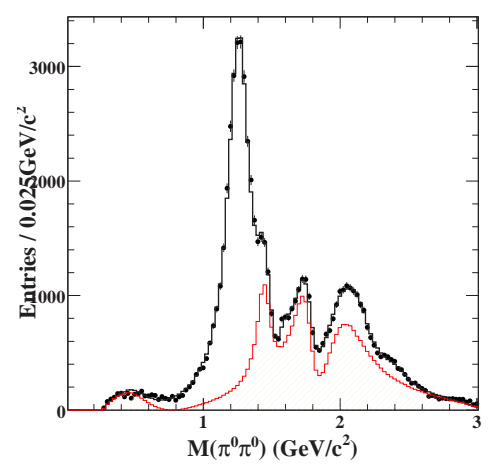

Figure 3: Comparisons between data and PWA fit projection. The black dots with error bars are data (with background subtracted), the solid histogram shows the PWA fit projection. The shaded histogram is for the contribution of $0^{++}$components.

The total scalar components contribution are displayed as shaded histogram in Fig. 3. There's 
clear evidence at low $\pi \pi$ invariant mass spectrum a broad structure due to the $f_{0}(500)$. The scalar states $f_{0}(1500), f_{0}(1710)$ and $f_{0}(2020)$ are also confirmed in the basic solution, each one with statistic significance larger than $10 \sigma$.

\section{4. $M(\omega \phi)$ threshold enhancement in $J / \psi \rightarrow \gamma \omega \phi$}

An anomalous near-threshold enhancement in the $\omega \phi$ invariant-mass spectrum in the process $J / \psi \rightarrow \gamma \omega \phi$ was reported by the BESII experiment [16]. A PWA that used a helicity covariant amplitude that assumed that the enhancement was produced by a resonance, denoted as the $X(1810)$, was performed on the BESII event sample. The analysis indicated that the $X(1810)$ favored $J^{P C}=0^{++}$over $J^{P C}=0^{-+}$or $2^{++}$with a significance of more than $10 \sigma$. The mass and width were determined to be $M=1812_{-26}^{+19}$ (stat) \pm 18 (syst) $\mathrm{MeV} / c^{2}$ and $\Gamma=105 \pm 20$ (stat) \pm 28 (syst) $\mathrm{MeV} / \mathrm{c}^{2}$, respectively, and the product branching fraction $\mathscr{B}(J / \psi \rightarrow \gamma X(1810) \rightarrow \gamma \omega \phi)=[2.61 \pm$ 0.27 (stat) \pm 0.65 (syst) $] \times 10^{-4}$ was measured. The $J / \psi \rightarrow \gamma \omega \phi$ decay mode is a doubly OZI suppressed process with a production rate that is expected to be suppressed relative to $J / \psi \rightarrow \gamma \omega \omega$ or $J / \psi \rightarrow \gamma \phi \phi$ by at least one order of magnitude [35]. Possible interpretations of the $\omega \phi$ threshold enhancement include a new type of resonance, such as a tetraquark state (with structure $q^{2} \bar{q}^{2}$ ) [36], a hybrid [37], or a glueball state [38] etc., a dynamical effect arising from intermediate meson rescattering [39], or a threshold cusp of an attracting resonance [40]. As of now none of these interpretations has either been established or ruled out by experiment.

A search for the $X(1810)$ was performed by the Belle collaboration in the decay of $B^{ \pm} \rightarrow$ $K^{ \pm} \omega \phi$ [41], but no obvious $X(1810)$ signal was observed. A high statistics data sample collected with the BESIII detector provides a good opportunity to confirm the existence of the $\omega \phi$ threshold enhancement, study its properties and search for other possible related states that decay to $\omega \phi$.

The doubly OZI suppressed decay modes $J / \psi \rightarrow \gamma \omega \phi\left(\omega \rightarrow \pi^{+} \pi^{-} \pi^{0}, \phi \rightarrow K^{+} K^{-}\right)$is studied based on the $225 \mathrm{M} J / \psi$ event sample accumulated with the BESIII detector [42]. The scatter plot of the $M_{K^{+} K^{-}}$versus $M_{\pi^{+} \pi^{-}} \pi^{0}$ invariant masses for events is shown in Fig. 4(a). A strong deviation from three-body $J / \psi \rightarrow \gamma \omega \phi$ phase space is observed near the $\omega \phi$ mass threshold in Fig $4(\mathrm{~b})$, which shows up as a diagonal band along the upper right-hand edge of the Dalitz plot in Fig 5 (a).

A partial wave analysis with a tensor covariant amplitude that assumes that the enhancement is due to the presence of a resonance, the $X(1810)$, is performed, and confirms that the spin-parity of the $X(1810)$ is $0^{++}$. The contributions of each component of the basic solution of the PWA fit are shown in Fig. 5(b). The mass and width of the $X(1810)$ are determined to be $M=1795 \pm$ 7 (stat) ${ }_{-5}^{+13}$ (syst) \pm 19 (mod) $\mathrm{MeV} / c^{2}$ and $\Gamma=95 \pm 10(\text { stat })_{-34}^{+21}($ syst $) \pm 75$ (mod) $\mathrm{MeV} / c^{2}$ and the product branching fraction is measured to be $\mathscr{B}(J / \psi \rightarrow \gamma X(1810) \rightarrow \gamma \omega \phi)=\left(2.00 \pm 0.08(\text { stat })_{-1.00}^{+0.45}(\right.$ syst $) \pm$ $1.30(\bmod )) \times 10^{-4}$. These results are consistent within errors with those of the BESII experiment [16].

The anomalous enhancement observed at the $\omega \phi$ invariant-mass threshold and the large measured branching fractions $(\sim 1 / 2$ of $\mathscr{B}(J / \psi \rightarrow \gamma \phi \phi)$ [29]) are surprising and interesting. The enhancement is not compatible with being due either to the $X(1835)$ or the $X(p \bar{p})$, due to the different mass and spin-parity. The interpretation of the enhancement as being due to effects of $\omega \phi$ final state interactions (FSI) is not excluded in this analysis. Searches for this structure in different decays modes, e.g. $K^{*} K^{*}, \omega \omega$, etc., and in other production processes, e.g. $J / \psi \rightarrow \phi \omega \phi, J / \psi \rightarrow \omega \omega \phi$ 

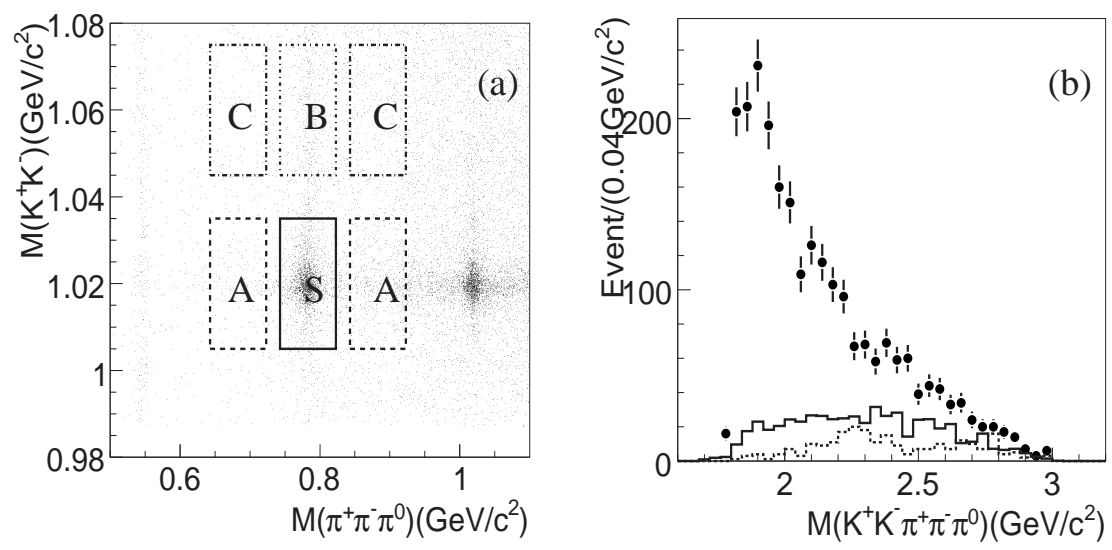

Figure 4: (a) A scatter plot of $M_{K^{+} K^{-}}$versus $M_{\pi^{+} \pi^{-}} \pi^{0}$. The boxes indicate the signal region labeled as $\mathrm{S}$ and sideband regions labeled as A, B and C. (b) the events in the $\omega \phi$ signal region; the solid histogram is the background distribution estimated from the sideband events, the dashed histogram is that obtained from inclusive $J / \psi$ MC samples.
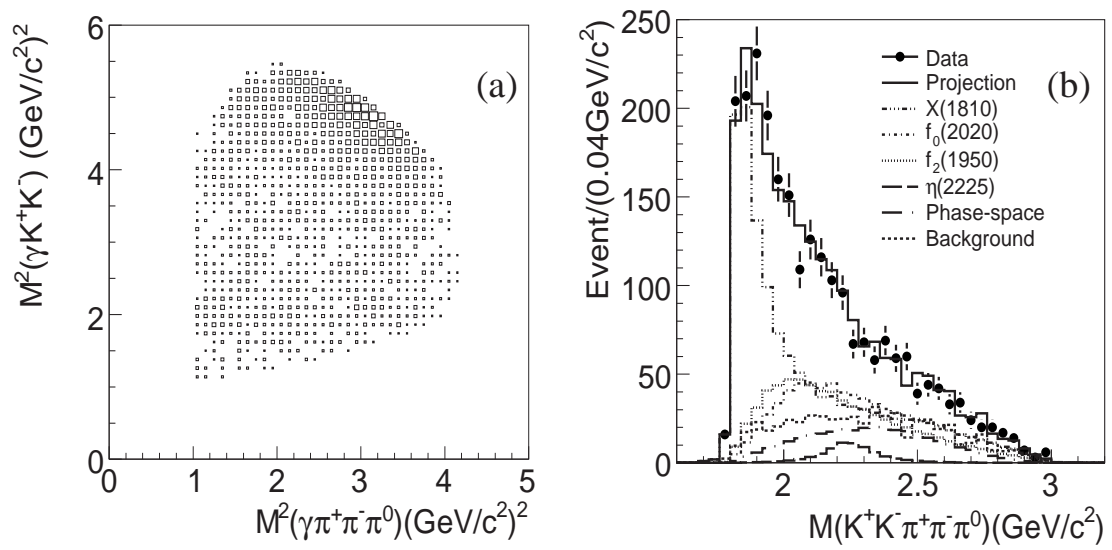

Figure 5: (a) A Dalitz plot of $M^{2}\left(\gamma \pi^{+} \pi^{-} \pi^{0}\right)$ versus $M^{2}\left(\gamma K^{+} K^{-}\right)$. (b) Comparisons between data and PWA fit projections for the $K^{+} K^{-} \pi^{+} \pi^{-} \pi^{0}$ invariant-mass distribution.

etc., are essential to explore the nature of the enhancement, and gain more insight in the underlying dynamics.

\section{Summary}

Based on 225M $J / \psi$ events collected by BESIII detector at BEPCII, the preliminary results from the partial wave analyses of $J / \psi \rightarrow \gamma \omega \phi, \gamma \pi^{0} \pi^{0}, \gamma \eta \eta$ are reported. A $0^{++}$state $X(1810)$, which was observed at BESII in the $\omega \phi$ mass spectrum in $J / \psi \rightarrow \gamma \omega \phi$, is confirmed at BESIII. The scalars $f_{0}(1500)$ and $f_{0}(1710)$ are observed and measured in both $J / \psi \rightarrow \gamma \eta \eta$ and $\gamma \pi^{0} \pi^{0}$ decays.

\section{Acknowledgments}

We thank the BEPCII group for excellent operation of the accelerator, the IHEP computer 
group for valuable computing and network support, and all the colleagues contributing on the physical measurements.

\section{References}

[1] F. E. Close, Rept. Prog. Phys. 51, 833 (1988).

[2] S. Godfrey and J. Napolitano, Rev. Mod. Phys. 71, 1411 (1999).

[3] C. Amsler and N. A. Tornqvist, Phys. Rept. 389, 61 (2004).

[4] E. Klempt and A. Zaitsev, Phys. Rept. 454, 1 (2007).

[5] V. Crede and C. A. Meyer, Prog. Part. Nucl. Phys. 63, 74 (2009).

[6] Y. Chen et al., Phys. Rev. D 73, 014516 (2006).

[7] E. Gregory et al., JHEP 1210, 170 (2012).

[8] C. Amsler et al. (Crystal Barrel Collaboration), Phys. Lett. B 340, 259 (1994).

[9] C. Amsler et al. (Crystal Barrel Collaboration), Phys. Lett. B 355, 425 (1995).

[10] J. Z. Bai et al. (BES Collaboration), Phys. Lett. B 472, 207 (2000).

[11] J. Z. Bai et al. (BES Collaboration), Phys. Rev. D 68, 052003 (2003).

[12] M. Ablikim et al. (BES Collaboration), Phys. Lett. B 642, 441 (2006).

[13] C. Amsler et al. [Crystal Barrel Collaboration], Phys. Lett. B 639, 165 (2006).

[14] M. Ablikim et al. (BES Collaboration), Phys. Lett. B 603, 138 (2004).

[15] I. Uman et al., Phys. Rev. D 73, 052009 (2006).

[16] M. Ablikim et al. (BES Collaboration), Phys. Rev. Lett. 96, 162002 (2006).

[17] M. Ablikim et al. (BESIII Collaboration), Nucl. Instrum. Meth. A 614, 345 (2010).

[18] M. Ablikim et al. (BESIII Collaboration), Chinese Phsyics C 36 (10), 915 (2012).

[19] C. Edwards et al., Phys. Rev. Lett. 48, 458 (1982).

[20] A. V. Anisovich et al., Phys. Lett. B 449, 145 (1999).

[21] A. V. Anisovich et al., Phys. Lett. B 491, 47 (2000).

[22] A. V. Anisovich et al., Nucl. Phys. A 662, 319 (2000).

[23] D. Barberis et al. (WA102 Collaboration), Phys. Lett. B 479, 59 (2000).

[24] C. Amsler et al. (Crystal Barrel Collaboration), Eur. Phys. J. C 23, 29 (2002).

[25] F. G. Binon et al., Phys. Atom. Nucl. 68, 960 (2005) [Yad. Fiz. 68, 998 (2005)].

[26] M. Ablikim et al. (BESIII Collaboration), arXiv:1301.0053.

[27] N. Berger, B. J. Liu and J. K. Wang, J. Phys. Conf. Ser. 219, 042031 (2010).

[28] B. S. Zou and D. V. Bugg, Eur. Phys. J. A 16, 537 (2003).

[29] J. Beringer et al. (Particle Data Group), Phys. Rev. D 86, 010001 (2012).

[30] R. M. Baltrusaities et al., Phys. Rev. D 35, 2077 (1987). 
[31] J. E. Augustin et al., Z. Phys. C 36, 369 (1987).

[32] J. Z. Bai et al., (BES Collaboration), Phys. Rev. Lett. 76, 3502 (1996).

[33] L. Kopke, N. Wermes, Phys. Rep. 174, 67 (1989).

[34] J. Z. Bai et al., (BES Collaboration), Phys. Rev. Lett. 81, 1179 (1998).

[35] Köpke L, Wermes N. J/ $\psi$ Decays. CERN-EP/88-93, Physics Reports 174 (1989) 67-227: CERN, CH-1211 Geneva 23, Switzerland.

[36] B. A. Li, Phys. Rev. D 74, 054017 (2006).

[37] K. T. Chao, arXiv:hep-ph/0602190.

[38] P. Bicudo et al., Eur. Phys. J. C 52, 363-374 (2007)

[39] Q. Zhao et al., Phys. Rev. D 74, 114025 (2006).

[40] D. V. Bugg, J. Phys. G 35, 075005 (2008)

[41] C. Liu et al. (Belle Collaboration), Phys. Rev. D 79, 0701102(R)(2009).

[42] M. Ablikim et al. (BESIII Collaboration), arXiv:1211.5668. 